\title{
Aplicação do método de Monte Carlo ao estudo de polímeros
}

\section{Resumo}

Discute-se a utilização do método de Monte Carlo na simulação de cadeias poliméricas. Destaca-se a importância de obtenção de informaçð̃es de natureza universal aplicáveis a qualquer polímero através da simulação numérica de polímeros modelos. Estabelece-se um paralelo entre as previsð̃es teóricas e os resultados de estudos Monte Carlo sobre polímeros em solução.

\section{Introdução}

O estudo de polímeros constitui um importante domínio de investigação no qual se incluem as fibras têxteis, os plásticos, celulose, proteínas e polinucleotídeos. O número de elementos que constituem uma cadeia de polímeros é da ordem de $10^{4}$. Devido ao grande número de configuraçōes que esta pode assumir, um estudo desses materiais não pode prescindir de um tratamento estatístico [1]. Na realidade, a uma cadeia polimérica constituída por $\mathrm{N}$ elos (ligaçőes entre os diversos elementos da cadeia), supondo-se que a cada elo estejam associadas $\mathrm{f}$ possibilidades rotacionais, corresponderão $\mathrm{f}^{\mathrm{N}}$ possiveis conformaçð̃es.

Diferentes metodologias foram estabelecidas no estudo de polímeros [2]. Por um lado privilegiou-se uma abordagem centrada sobre a constituição química dos elementos de uma cadeia e sobre a natureza covalente das ligaçōes estabelecidas entre suas unidades. Por outro lado, a necessidade de se aplicar um tratamento estatístico impôs que propriedades globais da cadeia fossem discutidas, em particular o seu aspecto geométrico. As propriedades geométricas de uma cadeia polimérica constituem portanto o principal objectivo de estudo de um tratamento estatístico [1].

A possibilidade de se utilizar computadores e neles simular através de experiências numéricas o comportamento de um sistema está na origem da aplicação do método de Monte Carlo [3] ao estudo de um grande número de sistemas, inclusive os polímeros. Este método, desenvolvido a partir das técnicas de cálculo numérico por geração de sequências de números aleatórios, transformou-se, paralelamente ao extraordinário desenvolvimento da tecnologia computacional, num poderoso método de investigação. As primeiras aplicaçðes do método de Monte Carlo ao estudo de polímeros foram realizadas nos anos cinquenta [4]. Embora as dimensðes e complexidade dos modelos estudados sejam actualmente maiores, o objectivo fundamental continua a ser o mesmo dos estudos precursores, isto é, privilegia-se a análise da estrutura geométrica das cadeias poliméricas, procurando-se extrair informaçðes globais e universais aplicáveis a um grande número de polímeros. É importante salientar que a grande maioria dos sistemas simulados são polímeros modelos nos quais podem ser omitidos detalhes sobre a estrutura química dos elementos da cadeia polimérica.

\section{As propriedades geométricas das cadeias poliméricas}

As propriedades geométricas de uma cadeia polimérica de $\mathrm{N}$ elos e $\mathrm{N}+1$ unidades são usualmente discutidas a partir de duas grandezas: a distância quadrática média entre os extremos da cadeia $\left\langle\mathrm{R}^{2}\right\rangle$ (Figura 1) e o raio de rotação quadrática médio $\left\langle\mathrm{S}^{2}\right\rangle$, definidos respectivamente da seguinte maneira:

$$
\begin{aligned}
& \left.\left\langle R^{2}\right\rangle=\left\langle\sum_{i=1}^{N} \vec{R}_{i} \cdot \vec{R}_{i}\right\rangle+2<\sum_{i \neq j} \vec{R}_{i} \cdot \vec{R}_{j}\right\rangle \\
& \left\langle S^{2}\right\rangle=\left\langle\left[\sum\left(\vec{R}_{i \neq j}-\vec{R}_{i}\right)^{2}\right] /(N+1)^{2}\right\rangle
\end{aligned}
$$

$\mathrm{O}$ raio quadrático médio $\left\langle\mathrm{R}^{2}\right\rangle$, também chamado de momento de distribuição de segunda ordem [1], carac-

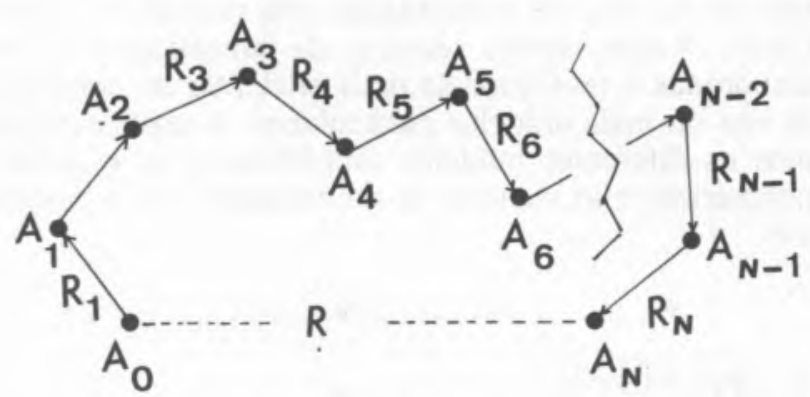

Figura 1

Representação em duas dimensð̄es de uma cadeia polimérica livre ou cadeia polimérica aleatoria

teriza a extensão da cadeia polimérica. Por sua vez, $\left\langle\mathrm{S}^{2}\right\rangle$ representa a distribuição da cadeia em torno de uma posição, definida geralmente como sendo o centro de gravidade do polímero. Essas propriedades são fundamentais para uma análise estrutural das cadeias e a sua obtenção para os diferentes modelos de estruturas poliméricas (Figura dois) constitue um dos principais objectivos.

Para uma cadeia hipotética, completamente livre, ou seja sem restriçð̃es (vínculos) aos possíveis graus de liberdade internos (cadeia polimérica aleatória), o ângulo $\theta$ entre os elos e o ângulo diédrico $\phi$ podem assumir

\footnotetext{
* Departamento de Química - Faculdade de Ciências de Lisboa
} 


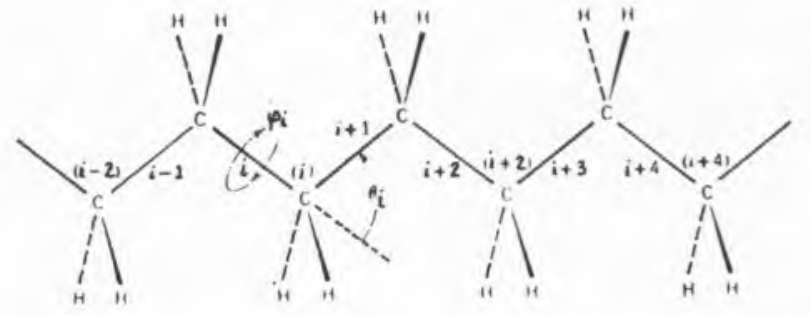

Figura 2

Representaçâo de um segmento de uma cadeia de polimetileno (Referência 1). De maneira geral, para polímeros modelos: (a) a cadeia é chamada de cadeia de rotação livre quando o ângulo entre os elos ( $\theta)$ é fixo, enquanto $\phi$ (ângulo entre os planos definidos pelos pares de elos (ligaçøes) $i-1, i$ e $i, i+1$ ) pode assumir qualquer valor. (b) a cadeia é chamada de livre ou aleatoria quando não houver restriçôes sobre os valores de $\theta$ e $\phi$

qualquer valor. Consequentemente, a segunda contribuição do lado direito de (1) é nula e neste caso:

$\left\langle\mathrm{R}^{2}>=\mathrm{NR}^{2}\right.$

Define-se então uma outra propriedade geométrica, chamada de "razão característica" [1] $\mathrm{C}_{\mathrm{N}}$, como:

$C_{N}=\left\langle R^{2}>/ N^{2}\right.$

\section{Simulação pelo método de Monte Carlo de políme- ros modelos}

Diferentes modelos simplificados para a simulação computacional de polímeros tem sido propostos [5] (Figura 3). Uma maior eficiência do processo de amostragem das possíveis configuraçðes de um modelo particular pode ser obtida através de uma variante bem adaptada da técnica de amostragem pelo método de Monte Carlo. Assim, várias técnicas de amostragem foram elaboradas e revelaram-se mais eficientes na simulação de um ou mais modelos particulares. A seguir, discutimos os diferentes modelos simplificados de estruturas poliméricas e as técnicas de amostragem a eles associados.

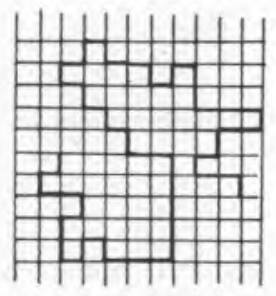

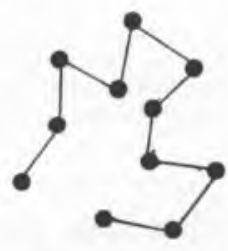

Figura 3
Modelos simplificados de cadeias poliméricas utilizados em simulaç̋es numéricas. (a) modelo de rede; (b) modelo de "linhas-e-botôes" ou modelo do "colar de pérolas"; (c) modelo de "molas-e-botð̃es". Extraída da referência [5]

$\mathrm{O}$ primeiro modelo (modelo de rede), proposto por $\mathrm{F}$. T. Wall e colaboradores [6] consistia simplesmente de uma rede (em duas ou três dimensø̃es) percorrida de maneira aleatória e única, isto é, nenhum sítio da rede pode ser visitado mais do que uma vez. Esta restrição visa satisfazer a condição de exclusão de volume existente num polimero real em consequência da forte interacção repulsiva a curta distância entre os vários ele- mentos da cadeia. Essa restrição ilustra a importância de recorrer-se ao método de Monte Carlo, já que a trajectória aleatória em duas dimensð̃es (Polya walk [3]) tinha uma solução analítica, mas a condição de exclusão de volume complicava enormemente uma abordagem deste tipo.

O modelo de rede é extremamente simples e permite simular através da geração de uma trajectória aleatória numa rede regular, o comportamento de uma cadeia polimérica flexível. Desta maneira, podem ser construídas diferentes configuraçðes, cada configuração correspondendo a uma trajectória aleatória através da rede. As quantidades médias de interesse, características geométricas, são então determinadas. Apesar de sua simplicidade, este método não é eficaz para modelos de cadeias poliméricas maiores do que um certo número de elementos $(N \geq 20)$. A razão essencial é a condição de exclusão de volume que obriga a interrupção da trajectória aleatória quando um mesmo sítio da rede for revisitado $[6,7]$.

Um outro modelo frequentemente utilizado é o modelo de "linha-e-botão" $[8,9,10]$ ou modelo do "colar de pérolas": constituído por $\mathrm{N}$ elos ou kinhas de ligação e por $\mathrm{N}+1$ "pérolas", associadas às junçð̌es entre os diferentes elos. Cada "pérola" pode ser representada por um potencial repulsivo $\mathrm{V}(\mathrm{r})$, o que permite satisfazer a condição de exclusão de volume. Uma das técnicas de amostragem estatística usualmente empregada para simular-se este modelo é chamada de "reptação" [11]. É uma técnica de amostragem considerada eficiente e pode ser aplicada a qualquer modelo de cadeia polimérica. Inicialmente, pode ser construída uma configuração arbitrária. Por exemplo, uma cadeia gerada aleatoriamente, mas que satisfaça as restriçøes para os valores de $\theta$ (ângulo entre elos) e $\phi$ (ângulo diédrico). Um dos dois extremos da cadeia é seleccionado de maneira aleatória, removido de sua posição inicial e transferido ao extremo oposto da cadeia ("reptação"). A orientação do elo na nova posição é também determinada de maneira aleatória e uma nova configuração é considerada como sendo gerada caso satisfaça a condição de exclusão de volume.

Uma técnica de amostragem usualmente utilizada pode ser chamada de técnica de perturbaçð̃es locais ("kink jump’”) [10]. Consiste na geração aleatória de perturbaçð̃es locais ao longo da cadeia polimérica. Por exemplo, um segmento da cadeia é seleccionado aleatoriamente e perturbado também de maneira aleatória pela aplicação de uma rotação num intervalo $[-\Delta \phi,+\Delta \phi]$. $\Delta \phi$ deve ser determinado "empiricamente" ao longo da experiência numérica de maneira que aproximadamente $50 \%$ das novas configuraçð̄es geradas, sejam aceites. Uma configuração "teste" pode ser recusada caso não satisfaça a condição de exclusão de volume. Um outro modelo de cadeias poliméricas é chamado de modelo de "molas-e-botðes" [22]. É constituído por N molas e $\mathrm{N}+1$ "'botðes". Cada mola é representada por um potencial harmónico do tipo:

$$
\begin{array}{rlrl}
\mathrm{V}(\mathrm{r}) & =-\mathrm{V}_{\mathrm{o}} \ln \left[1-\left(\mathrm{r} / \mathrm{r}_{\mathrm{o}}\right)^{2}\right] ; & \mathrm{r}_{\mathrm{o}} \geq \mathrm{r} \\
& =0 \quad ; \mathrm{r}_{\mathrm{o}}<\mathrm{r}
\end{array}
$$

onde $V_{0}$ e $r_{0}$ são constantes. A condição'de exclusão de volume é såtisfeita associando-se a cada "botão" um potencial repulsivo. Este modelo foi simulado pela técnica de amostragem de reptação [11], mas informaçð̃es 
sobre propriedades dinâmicas da cadeia polimérica podem ser obtidas através do método de dinâmica Browniana $[13,14]$.

\section{Previsões teóricas e experimentais versus simulações Monte Carlo}

Um dos principais objectivos das experiências numéricas consiste na verificação de previsð̃es teóricas, algumas de difícil comprovação experimental. Por outro lado, os modelos teóricos são algumas vezes extremamente simplificados e a comparação com os resultados de uma experiência clássica real, é de validade limitada. $\mathrm{Na}$ realidade, uma característica importante das "experiências numéricas" é que elas permitem que se discuta um modelo teórico bem preciso. Neste espírito, apresentamos a seguir um resumo dos resultados mais significativos de experiências numéricas de modelos simplificados de polímeros através do método de Monte Carlo. Esssas simulaçб̃es permitiram elucidar algumas questðes sobre o comportamento de cadeias poliméricas.

\section{Simulação de uma cadeia polimérica a diluição infinita}

Uma das previsð̌es da teoria de Flory [1] e de outras abordagens analíticas, verificada pela simulação de polímeros modelos, é relativa à distância quadrática média entre os extremos de uma cadeia polimérica no limite de $\mathrm{N} \rightarrow \infty$; limite assimptótico que parece ser atingido numa experiência numérica para $\mathrm{N} \cong 100$ [12]:

$$
\left\langle\mathrm{R}^{2}>=\mathrm{AN}^{2 \mu}\right.
$$

onde A é uma constante, função da temperatura e das propriedades do solvente. $\mathrm{O}$ valor do expoente $\mu$ é universal e aplica-se a qualquer cadeia polimérica. Depende da dimensionalidade do sistema. $\mu=0.6$ em três dimensð̃es e $\mu=0.7 \mathrm{em}$ duas dimensð̋es. É importante observar que para um polímero a diluição infinita, onde as interacçð̋es entre elementos de diferentes cadeias podem ser ignoradas, a diferença entre os dois expoentes relaciona-se unicamente com o "efeito de exclusão de volume" que é evidentemente mais importante a duas dimensठ̄es. A relação (6) foi verificada por várias simulaçðes Monte Carlo de polímeros modelos $[6,10,15,16]$.

\section{Simulação do efeito de solvente sobre a estrutura de uma cadeia polimérica}

Um fenómeno de importância e que permitiu comparar as previsð̌es teóricas de Flory $[1,2]$ com resultados de experiências numéricas, relaciona-se com o comportamento de um polimero em solução na qual a interacção com o solvente pode exercer um papel importante sobre a estruturação da cadeia polimérica. Apesar do papel desempenhado pelo efeito de exclusão de volume (interacçðes intramoleculares), as interacçð̄es entre elementos da cadeia polimérica e o solvente (intermoleculares) podem contribuir para que o polímero se contraia (solvente pobre) ou se expanda (solvente super-perfeito). A alteração da estrutura do polímero é relativa à forma que assume a diluição infinita num solvente perfeito no qual somente o efeito de exclusão de volume é considerado.

Essas influências (solvente pobre e solvente super-perfeito) podem ser modeladas numa simulação de Monte Carlo através da introdução de um potencial efectivo entre os diversos elementos da cadeia polimérica $[17,18,19]$. O potencial efectivo é constituído por uma parte repulsiva e por outra atractiva. Sabe-se que a parte atractiva de um potencial de interacção é mais importante em temperaturas mais baixas e que a parte repulsiva é mais importante a temperaturas mais altas. Assim, através da variação de temperatura pode-se simular um solvente super-perfeito (alta temperatura) e um solvente pobre (baixa temperatura). A simulação deste feito de solvente pela introdução de um potencial efectivo permitiu verificar a validade da teoria de Flory $[1,2]$ no regime de exclusão de volume onde as interacçðеs intramoleculares exercem o papel mais importante. Um fenómeno interessante, reproduzido por simulaçбes numéricas, [19] é a situação chamada de "colapso" da cadeia polimérica: as interacçø̃es fortemente repulsivas da cadeia com o solvente transformam o polimero numa estrutura globular compacta (Figura 4).

\section{Simulação de cadeias poliméricas múltiplas}

A importância que assume a utilização do método de Monte Carlo no estudo de sistemas poliméricos é parti-
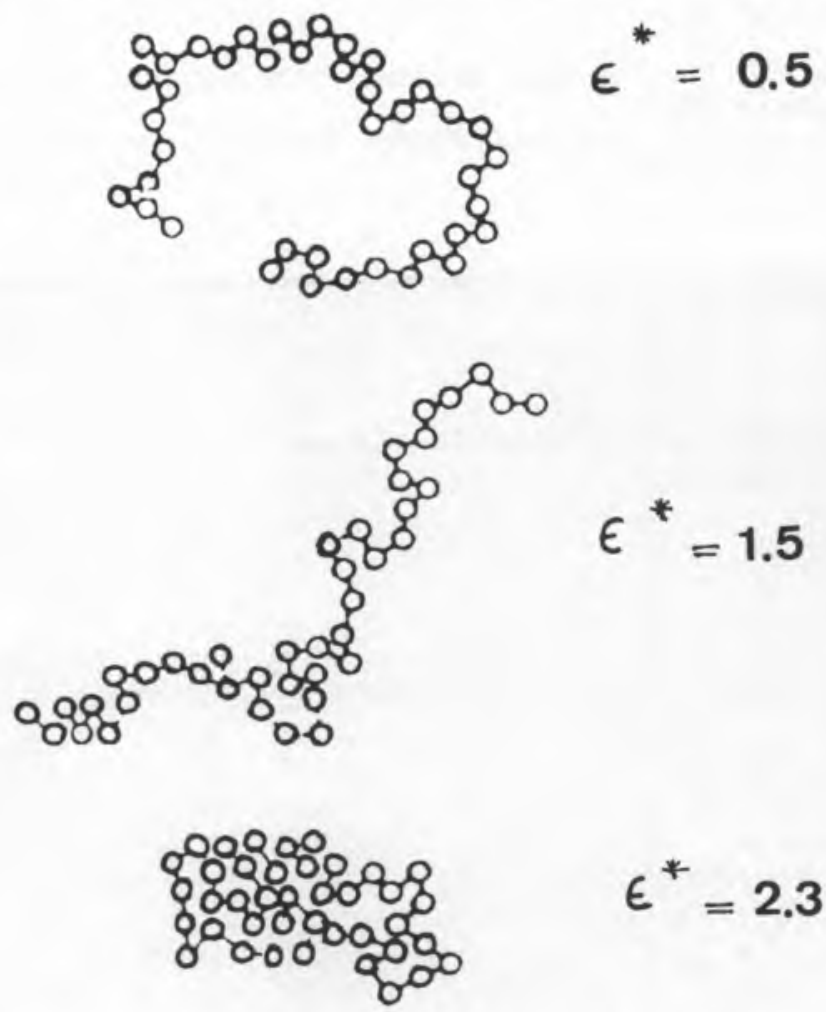

$$
\epsilon^{*}=2.3
$$

Figura 4

Resultados da simulação da referência [19] mostrando um instantâneo de uma cadeia polimérica em solução para três valores da temperatura: $\epsilon^{*}=\epsilon / k T$; para $\epsilon^{*}=2.3$ verifica-se a situação chamada de colapso da cadeia polimérica

cularmente bem ilustrada na simulação de cadeias poliméricas múltiplas $[20,21]$. Esses estudos permitem discutir o efeito da densidade e das interacçס̃es intramoleculares e intermoleculares sobre a estruturação de uma cadeia polimérica. $\mathrm{O}$ estudo de cadeias poliméricas múltiplas é muito mais complexo que o de uma cadeia isolada. Diferentes teorias analíticas para cadeias poliméricas múltiplas foram propostas: Flory e Huggins [2] propuseram um modelo de rede, Fixman [22] um modelo de potencial efectivo dependente apenas da distância entre os centros de massa de uma cadeia polimérica. Prigogine e colaboradores [23] propuseram um modelo chamado de celular. Nesse contexto, as simulaçðes numéricas representaram também uma verificação do 
fundamento dessas teorias e das aproximaçסes que lhe são inerentes. Um resultado interessante de simulaçð̃es numéricas de cadeias poliméricas múltiplas pelo método de Monte Carlo [20] foi a verificação da existência de uma situação chamada de ponto $\theta$ (theta), previsto por Flory $[1,2]$. Neste ponto, as forças intramoleculares e intermoleculares contrabalançam-se e a cadeia polimérica comporta-se como uma cadeia polimérica completamente aleatória ou cadeia polimérica livre.

\section{Conclusões}

A realização de experiências numéricas em computadores pelo método de Monte Carlo contribuiu de maneira significativa para a compreensão da estrutura de cadeias poliméricas e permitiu verificar o fundamento de teorias analíticas. Os métodos de simulação numérica representam actualmente um instrumento de investigação importante, fornecendo informaçð̄es complementares às abordagens teóricas e experimental.

\section{REFERÊNCIAS}

[1] P. J. Flory, Statistical Mechanics of Chain Molecules. (John Wiley - 1969)

[2] P. J. Flory, Principles of Polymer Chemistry. (Cornell University Press - 1953).
[3] Hammersley e Handscomb, Monte Carlo Methods. (Chapman and Hall -1979$)$.

[4] F. T. Wall, J. Chem. Phys., 21, 1914 (1953). E. W. Montroll, J. Chem. Phys., 18, 734 (1950). F. T. Wall, R. J. Rubin e L. M. Isaacson, J. Chem. Phys., 22,1036 (1955).

[5] A. Baumgärtner, Topics in Current Physics, vol. 36, Capítulo 5.

[6] F. T. Wall e J. J. Erpenbeck, J. Chem. Phys., 30,634 (1959).

[7] M. N. Rosenbluth e A. W. Rosenbluth, J. Chem. Phys., 23,356 (1955).

[8] R. Grishman, J. Chem. Phys., 58,220 (1973).

[9] W. Bruns, J. Phys. A10, 1963 (1977).

[10] A. Baumgärtner e K. Binder, J. Chem. Phys., 71,2541 (1979).

[11] F. T. Wall e F. Mandel, J. Chem. Phys., 63, 4592 (1975).

[12] M. Bishop, D. Ceperley, M. H. Kalos e H. L. Frisch, J. Chem. Phys., 70, 1299 (1979).

[13] D. Cerpeley, M. H. Kalos e J. L. Lebowitz, Phys. Rev. Lett., 41, 313 (1978).

[14] R. C. Armstrong. J. Chem. Phys., 63, 4592 (1975).

[15] M. Bishop e J. P. J. Michels, J. Chem. Phys., 82, 1059 (1985).

[16] M. Bishop e J. P. J. Michels, J. Chem. Phys., 83,4791 (1985).

[17] M. E. Fisher e B. J. Hiley, J. Chem. Phys., 39, 1253 (1961).

[18] P. H. Verdier e W. H. Stockmayer, J. Chem. Phys., 36,227 (1962).

[19] J. Tobochnik, I. Webman, J. L. Lebowitz e M. H. Kalos, Macromolecules, 15, 549 (1982)

[20] J. G. Curro, J. Chem. Phys., 61, 1203 (1974).

[21] M. Bishop, D. Ceperley, H. L. Frisch e M. H. Kalos, J. Chem. Phys., 72,3228 (1980).

[22] M. Fixman, J. Chem. Phys., 33, 370 (1960). M. Fixman e J. M. Peterson, J. Am. Chem. Soc., 3524 (1964).

[23] I. Prigogine, A. Bellemans e V. Mathot, The Molecular Theory of Solutions (Interscience, New York-1957).

\section{AD HOC}

\section{A reunião extraordinária da SCRATCH}

O Tac responsável pela coordenação da SCRATCH (Sociedade Cooperativa e Regional de Apoio aos Tacs com Curricula Hinquietantes) marcou o início dos trabatho com um olhar cansado mas firme sobre a ainda turbulenta assembleia dos Tacs, expressamente convocada para deliberar sobre as atitudes a tomar face a uma exigência de discussão pública das actividades dos seus membros no último quinquénio.

- Começo por afirmar a minha abstenção com respeito a esta imposição da Lei. As razðes são óbvias: Não quero que alguém pense, em particular os Saps e os Xuas, que nós recusamos porque nada fizemos. Pela minha parte, tenho a consciência bem tranquila. Sete dos oito Xuas e Saps do meu grupo foram, por minha influência directa, para o estrangeiro, tendo obtido pieisdis. O oitavo é hoje Sap graças à inestimável colaboração que me tem dado, nos últimos 23 anos, na formaçăo de Gnes. Maugrado tudo isto, tenho consciência que alguns dos presentes poderâo estar em situação hinquietante.

Pediu então a palavra um Tac bastante mais novo, que, cofiando a barba e limpando os óculos embaciados, disse:

- Trabalhei duramente para, no período de 4 meses e meio após ter adquirido um pieisdi, obter $\mathrm{o}$ grau de incorporado. Saibam V. Exas. que apresentei 6 comunicaçðes ao Congresso Anual dos Gnes, quatro das quais foram publicadas nos "Procedimentos". Além disso, fui co-autor de 2 artigos, publicados num semanário de grande expansão, sob o título global " $\mathrm{Da}$ constituição de Júris de Pieisdi e de Incorporação - Como Bem Implementar a Hierarquia da Competência e Vice-Versa", os quais serviram de base à lei que hoje vigora. Nos últimos cinco anos, caros confrades, desenvolvi um projecto de colaboração com a Companhia Distribuidora de Leite com vista a uma racionalização da forma $e$ materiais da embalagem. Esta investigação, cujo financiamento foi incondicionalmente apoiado pelas entidades oficiais e pela própria empresa, em regime de "ventura junta", dado o seu carácter puro e, simultaneamente, aplicado, viu recentemente, e uma vez mais, o reconhecimento público, ao mais alto nivel, na recepção oferecida (Vossas Excelências sabem por quem) em Outubro passado, Por tudo isto, venho manifestar a minha concordância com 0 colega Tac Coordenador: Abstenho-me porque, sem ter "glass roofs", alguns dos presentes poderão estar em situação hinquietante.

- Após duas intervençðes onde a posição foi de abstenção, parece-me que podemos poupar o nosso precioso tempo perguntando se há aqui algum colega que esteja hinquieto. A estas palavras' sintéticas do Tac Coordenador seguiu-se um leve burburinho na sala. Trocas de olhares, acenos, abanar de cabeças. Ninguém se sentia hinquieto. Todos os Tacs se abstinham. Com a gravidade que $o$ assunto the impunha e a solenidade que 0 momento clamava, retomou então a palavra o Tac Coordenador:

- Fica assim decidido que vamos mostrar à evidência quem somos. Todos temos consciência do inestimável valor da nossa actividade. Não há razão para hinquietaçðes. Estava terminada a reunião extraordinária da SCRATCH.

Reportagem de José de Sousa 